\title{
Crystal structure of 6,6'-((1E, $\left.1^{\prime} E\right)-(((1 R, 2 R)-$ 1,2-diphenylethane-1,2-diyl) bis(azanylylidene)) bis(methanylylidene))bis(2-ethylphenol), $\mathrm{C}_{32} \mathrm{H}_{32} \mathrm{~N}_{2} \mathrm{O}_{2}$
}

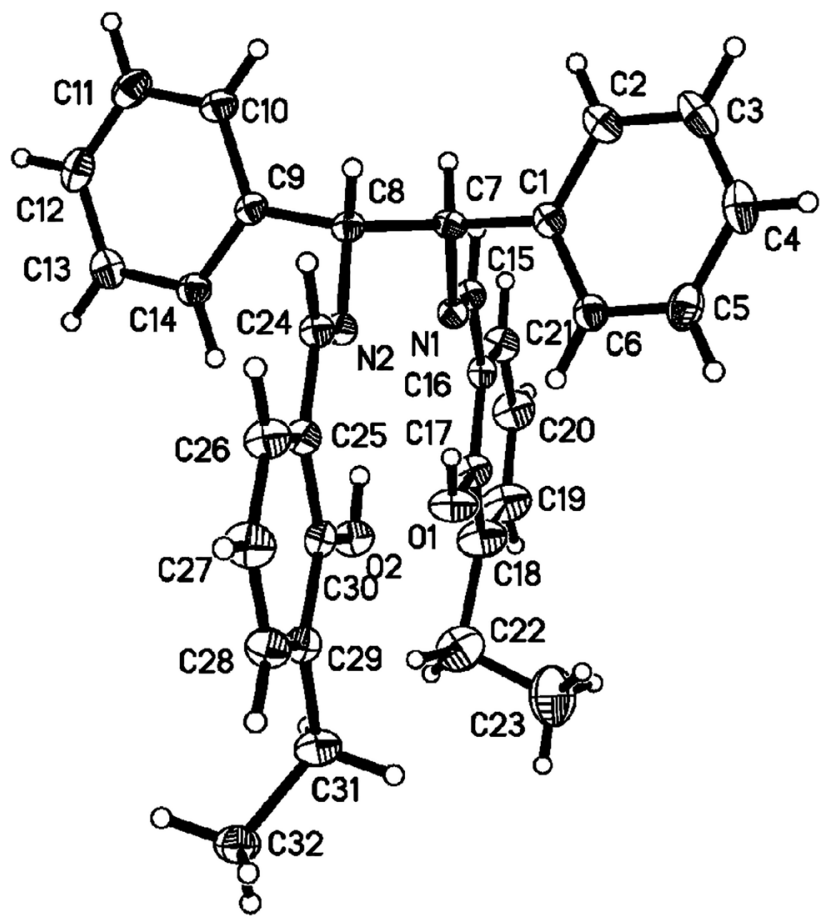

https://doi.org/10.1515/ncrs-2021-0060

Received February 10, 2021; accepted February 26, 2021; published online March 12, 2021

\section{Abstract \\ $\mathrm{C}_{32} \mathrm{H}_{32} \mathrm{~N}_{2} \mathrm{O}_{2}$, orthorhombic, $P 2_{1} 2_{1} 2_{1}$ (no. 19), $a=9.78920(12) \AA$, $b=12.71913(14) \AA, c=20.9423(3) \AA, V=2607.53(5)$ $\AA^{3}, Z=4, R_{g t}(\mathrm{~F})=0.0535, w R_{r e f}\left(F^{2}\right)=0.1365$, $T=149.99(10) \mathrm{K}$.}

CCDC no.: 2052199

\footnotetext{
*Corresponding author: Da-Bin Shi, School of Pharmaceutical Sciences, Zunyi Medical University, Zunyi 563000, P. R. China, E-mail: sdb007.student@sina.com. https://orcid.org/0000-00022787-9524

Zeng-Bing Xu and Jing-Yuan He, School of Pharmaceutical Sciences, Zunyi Medical University, Zunyi 563000, P. R. China
}

Table 1: Data collection and handling.

\begin{tabular}{|c|c|}
\hline Crystal: & Yellow block \\
\hline Size: & $0.13 \times 0.12 \times 0.10 \mathrm{~mm}$ \\
\hline Wavelength: & 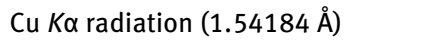 \\
\hline$\mu:$ & $0.59 \mathrm{~mm}^{-1}$ \\
\hline Diffractometer, scan mode: & SuperNova, $\omega$ \\
\hline$\theta_{\max }$, completeness: & $73.8^{\circ},>99 \%$ \\
\hline$N(h k l)_{\text {measured }}, N(h k l)_{\text {unique }}, R_{\text {int }}:$ & $12,561,5133,0.024$ \\
\hline Criterion for $I_{\mathrm{obs}}, N(h k l)_{\mathrm{gt}}:$ & $I_{\text {obs }}>2 \sigma\left(I_{\text {obs }}\right), 5044$ \\
\hline$N(\text { param })_{\text {refined: }}$ & 329 \\
\hline Programs: & CrysAlis $^{\mathrm{PRO}}[1]$, SHELX $[2,3]$, Olex2 [4] \\
\hline
\end{tabular}

The molecular structure is shown in the figure. Table 1 contains crystallographic data and Table 2 contains the list of the atoms including atomic coordinates and displacement parameters.

\section{Source of material}

A solution of 2-ethylphenol (2.4 g, $20 \mathrm{mmol}), \mathrm{MgCl}_{2}$ $(5.7 \mathrm{~g}, 60 \mathrm{mmol})$, paraformaldehyde $(9.0 \mathrm{~g}, 0.1 \mathrm{~mol})$, triethylamine (15 g $149 \mathrm{mmol})$ in acetonitrile $(50 \mathrm{~mL})$ was refluxed until full consumption of 2-ethylphenol (as indicated by TLC). The mixture was quenched with $\mathrm{HCl}(1 \mathrm{M})$ and extracted with ethyl acetate. The combined organic layers were washed with brine, dried with $\mathrm{MgSO}_{4}$ and evaporated under reduced pressure. The crude residue was purified by column chromatography to obtain 3-ethyl-2-hydroxybenzaldehyde (2.2 g, 73\% yield) as a yellow oil.

Synthesis of the title compound [5]: 3-ethyl-2hydroxybenzaldehyde ( $1 \mathrm{~g}, 6.7 \mathrm{mmol})$ was dissolved in $30 \mathrm{~mL}$ of ethanol and $(1 R, 2 R)-(-)-1,2$-diphenylethane1,2-diamine ( $0.71 \mathrm{~g}, 3.35 \mathrm{mmol})$ was added to the solution. The solution was heated to reflux for $4 \mathrm{~h}$. The solvent was removed in vacuo and $1.48 \mathrm{~g}$ of the product was obtained as yellow solid ( $95 \%$ yield). Crystals were obtained by slow evaporation of an ethanol solution at room temperature over a period of seven days, yield: $0.72 \mathrm{~g}(93 \%)$. M.p.: $105-107{ }^{\circ} \mathrm{C}$. Elemental analysis - found: C, 
Table 2: Fractional atomic coordinates and isotropic or equivalent isotropic displacement parameters $\left(\AA^{2}\right)$.

\begin{tabular}{|c|c|c|c|c|}
\hline Atom & $x$ & $y$ & $z$ & $U_{\text {iso }} * / U_{\text {eq }}$ \\
\hline 01 & $0.6789(3)$ & $0.32760(18)$ & $0.83208(10)$ & $0.0439(6)$ \\
\hline $\mathrm{H} 1$ & 0.639420 & 0.351668 & 0.800779 & $0.066^{*}$ \\
\hline 02 & $0.7564(2)$ & $0.24223(15)$ & $0.68175(9)$ & $0.0322(4)$ \\
\hline $\mathrm{H} 2$ & 0.713378 & 0.297399 & 0.684240 & $0.048^{*}$ \\
\hline N1 & $0.5336(2)$ & $0.46340(18)$ & $0.76594(10)$ & $0.0246(5)$ \\
\hline N2 & $0.6306(2)$ & $0.40912(18)$ & $0.64151(10)$ & $0.0256(5)$ \\
\hline C1 & $0.3444(3)$ & $0.4322(2)$ & 0.69217 (12) & $0.0273(6)$ \\
\hline $\mathrm{C} 2$ & $0.2207(3)$ & $0.4771(3)$ & $0.67516(14)$ & $0.0353(6)$ \\
\hline $\mathrm{H} 2 \mathrm{~A}$ & 0.211018 & 0.549854 & 0.675520 & $0.042^{*}$ \\
\hline $\mathrm{C} 3$ & $0.1104(3)$ & $0.4138(3)$ & $0.65749(16)$ & $0.0445(8)$ \\
\hline H3 & 0.027836 & 0.444509 & 0.645943 & $0.053^{*}$ \\
\hline $\mathrm{C} 4$ & $0.1236(3)$ & $0.3059(3)$ & $0.65709(16)$ & $0.0441(8)$ \\
\hline $\mathrm{H} 4$ & 0.049815 & 0.264019 & 0.645477 & $0.053^{*}$ \\
\hline $\mathrm{C} 5$ & $0.2459(3)$ & 0.2599 (3) & $0.67386(15)$ & $0.0404(7)$ \\
\hline H5 & 0.254620 & 0.187130 & 0.673122 & $0.048^{*}$ \\
\hline C6 & $0.3562(3)$ & $0.3220(2)$ & $0.69188(13)$ & $0.0316(6)$ \\
\hline $\mathrm{H} 6$ & 0.438045 & 0.290510 & 0.703796 & $0.038^{*}$ \\
\hline $\mathrm{C} 7$ & $0.4638(3)$ & $0.5024(2)$ & $0.70887(12)$ & $0.0260(5)$ \\
\hline $\mathrm{H} 7$ & 0.428554 & 0.572972 & 0.718036 & $0.031^{*}$ \\
\hline $\mathrm{C} 8$ & $0.5647(3)$ & $0.5104(2)$ & $0.65175(12)$ & $0.0250(5)$ \\
\hline $\mathrm{H} 8$ & 0.511601 & 0.527226 & 0.613415 & $0.030^{*}$ \\
\hline C9 & $0.6702(3)$ & $0.5964(2)$ & $0.66101(12)$ & $0.0258(5)$ \\
\hline C10 & $0.6379(3)$ & $0.6985(2)$ & $0.64190(14)$ & $0.0338(6)$ \\
\hline $\mathrm{H} 10$ & 0.551544 & 0.713166 & 0.625636 & $0.041^{*}$ \\
\hline C11 & $0.7340(4)$ & $0.7783(2)$ & $0.64705(16)$ & $0.0426(8)$ \\
\hline H11 & 0.712019 & 0.846188 & 0.634143 & $0.051^{*}$ \\
\hline $\mathrm{C} 12$ & $0.8614(4)$ & $0.7572(3)$ & $0.67116(15)$ & $0.0421(7)$ \\
\hline $\mathrm{H} 12$ & 0.925849 & 0.810646 & 0.674337 & $0.051^{*}$ \\
\hline C13 & $0.8941(3)$ & $0.6565(3)$ & $0.69071(15)$ & $0.0392(7)$ \\
\hline $\mathrm{H} 13$ & 0.980550 & 0.64 & 7092 & $0.047^{\star}$ \\
\hline C14 & $0.7985(3)$ & $0.5762(2)$ & $0.68602(13)$ & $0.0314(6)$ \\
\hline H14 & 0.820708 & 0.508760 & 0.699676 & $0.038^{*}$ \\
\hline $\mathrm{C} 15$ & $0.5159(3)$ & $0.5141(2)$ & $0.81800(13)$ & $0.0262(5)$ \\
\hline $\mathrm{H} 15$ & 0.465318 & 0.575987 & 0.816969 & $0.031^{*}$ \\
\hline $\mathrm{C} 16$ & $0.5716(3)$ & $0.4789(2)$ & $0.87885(12)$ & $0.0266(5)$ \\
\hline $\mathrm{C} 17$ & $0.6504(3)$ & $0.3873(2)$ & $0.88375(13)$ & $0.0344(6)$ \\
\hline C18 & $0.7057(5)$ & $0.3569(3)$ & $0.94287(17)$ & $0.0556(9)$ \\
\hline C19 & $0.6700(5)$ & $0.4170(3)$ & $0.99639(16)$ & $0.0583(11)$ \\
\hline H19 & 0.699968 & & 1.036385 & $0.070^{*}$ \\
\hline $\mathrm{C} 20$ & $0.5925(4)$ & $0.5070(3)$ & $0.99227(15)$ & $0.0464(8)$ \\
\hline $\mathrm{H} 20$ & 0.572640 & 0.545837 & 1.028721 & $0.056^{*}$ \\
\hline $\mathrm{C} 21$ & $0.5447(3)$ & $0.5388(3)$ & 0.93344 (14) & $0.0356(6)$ \\
\hline $\mathrm{H} 21$ & 0.494133 & 0.600447 & 0.930036 & $0.043^{*}$ \\
\hline $\mathrm{C} 22$ & $0.8091(5)$ & $0.2626(4)$ & $0.9480(2)$ & $0.0645(10)$ \\
\hline $\mathrm{H} 22 \mathrm{~A}$ & 0.855318 & 0.251761 & 0.907563 & $0.077^{\star}$ \\
\hline $\mathrm{H} 22 \mathrm{~B}$ & 0.877170 & 0.276721 & 0.980547 & $0.077^{\star}$ \\
\hline $\mathrm{C} 23$ & $0.7290(6)$ & $0.1703(5)$ & 0.9649 (3) & $0.0855(16)$ \\
\hline $\mathrm{H} 23 \mathrm{~A}$ & 0.663891 & 0.156148 & 0.931702 & $0.128^{*}$ \\
\hline $\mathrm{H} 23 \mathrm{~B}$ & 0.681683 & 0.182998 & 1.004286 & $0.128^{*}$ \\
\hline $\mathrm{H} 23 \mathrm{C}$ & 0.788601 & 0.110925 & 0.969793 & $0.128^{*}$ \\
\hline C24 & $0.6364(3)$ & $0.3722(2)$ & $0.58495(12)$ & $0.0270(5)$ \\
\hline $\mathrm{H} 24$ & 0.595413 & 0.409393 & 0.551853 & $0.032^{*}$ \\
\hline $\mathrm{C} 25$ & $0.7054(3)$ & $0.2731(2)$ & $0.57068(12)$ & $0.0268(5)$ \\
\hline $\mathrm{C} 26$ & $0.7155(4)$ & $0.2385(2)$ & $0.50772(13)$ & $0.0360(7)$ \\
\hline H26 & 0.675961 & 0.277889 & 0.475200 & $0.043^{*}$ \\
\hline
\end{tabular}

Table 2: (continued)

\begin{tabular}{lrrrr}
\hline Atom & $\boldsymbol{x}$ & $\boldsymbol{y}$ & $\boldsymbol{z}$ & $\boldsymbol{U}_{\text {iso }}{ }^{*} / \boldsymbol{U}_{\text {eq }}$ \\
\hline $\mathrm{C} 27$ & $0.7833(4)$ & $0.1468(3)$ & $0.49300(15)$ & $0.0422(8)$ \\
$\mathrm{H} 27$ & 0.789439 & 0.124098 & 0.450882 & $0.051^{*}$ \\
$\mathrm{C} 28$ & $0.8424(3)$ & $0.0887(2)$ & $0.54176(15)$ & $0.0364(7)$ \\
$\mathrm{H} 28$ & 0.888045 & 0.026869 & 0.531477 & $0.044^{*}$ \\
$\mathrm{C} 29$ & $0.8360(3)$ & $0.1193(2)$ & $0.60534(13)$ & $0.0290(6)$ \\
C30 & $0.7651(3)$ & $0.2123(2)$ & $0.61966(12)$ & $0.0256(5)$ \\
C31 & $0.9035(4)$ & $0.0582(3)$ & $0.65828(16)$ & $0.0396(7)$ \\
H31A & 0.840309 & 0.004905 & 0.673357 & $0.048^{*}$ \\
H31B & 0.921679 & 0.105529 & 0.693570 & $0.048^{*}$ \\
C32 & $1.0362(4)$ & $0.0051(3)$ & $0.6391(2)$ & $0.0479(8)$ \\
H32A & 1.018357 & -0.045495 & 0.606058 & $0.072^{*}$ \\
H32B & 1.074920 & -0.029840 & 0.675442 & $0.072^{*}$ \\
H32C & 1.099145 & 0.056967 & 0.623522 & $0.072^{*}$ \\
\hline \multicolumn{5}{c}{} \\
80.68\%; H, 6.72\%; N, 5.84\%; calculated for $\mathrm{C}_{32} \mathrm{H}_{32} \mathrm{~N}_{2} \mathrm{O}_{2}$ : C, \\
80.64\%; H, 6.77\%; N, 5.88\%.
\end{tabular}

Data were collected via CrysAlis ${ }^{\mathrm{PRO}} 1.171 .39 .7 \mathrm{e}$ [1], the structure of crystal was determined by SHELXT [2] and refined by OLEX2 [4] and SHELXL [3].

The absolute structure determination succeeded as the derived Flack parameter is found to be near zero with a low standard uncertainty [-0.02(9) from 2090 selected quotients] using Parsons' method [6]. There is a small disorder of one ethyl group (C22, C23) which was not included in the refinement.

\section{Comment}

Hugo Schiff described the condensation between an aldehyde and an amine leading to a Schiff base in 1864 [7]. Schiff base ligands are privileged ligands, as they are easily prepared by the condensation between aldehydes and imines. Stereogenic centres can be introduced in the synthetic design. Schiff base ligands are able to coordinate many different metals, and to stabilize them in various oxidation states, enabling the use of Schiff base metal complexes for a large variety of useful catalytic transformations [8-10]. When two equivalents of salicylaldehyde are combined with a diamine, a particular chelating Schiff base is obtained known as salen - with four coordinating sites and two axial sites open to ancillary ligands. Here, we report a new chiral salen compound.

The asymmetric unit of the title structure contains one $6,6^{\prime}-\left(\left(1 E, 1^{\prime} E\right)-(((1 R, 2 R)-1,2\right.$-diphenylethane-1,2-diyl $)$ 
bis(azanylylidene))bis(methanylylidene))bis(2-ethylphenol). In the crystal structure, bond lengths and bond angles within the molecule are in agreement with the values reported [11]. The bond lengths of $\mathrm{C} 15-\mathrm{N} 1$ and $\mathrm{C} 24-\mathrm{N} 2$ are 1.278(3) and 1.275(3) $\AA$, respectively. As a result of the conjugation of the benzene moiety and adjacent carbonnitrogen double bond, the bond lengths of $\mathrm{C} 15-\mathrm{C} 16$ and C24-C25 are 1.457(4) and 1.461(4) A respectively, which is shorter than that of typical $\mathrm{C}-\mathrm{C}$ bond. Furthermore, the bond lengths of $\mathrm{C} 17-01$ and $\mathrm{C} 30-02$ are 1.351(3) and 1.358(3) A, respectively. The bond angles (C15-N1-C7) and $(\mathrm{C} 24-\mathrm{N} 2-\mathrm{C} 8)$ are $117.5(2)^{\circ}$ and $118.8(2)^{\circ}$, respectively. There are two intramolecular hydrogen bonds $(d \mathrm{D} \cdots \mathrm{A} 2.63 \AA$; $\mathrm{H} \cdots \mathrm{A} 1.904 \AA$ A ) between $\mathrm{H} 1$ and $\mathrm{N} 1$ atom and $(d \mathrm{D} \cdots \mathrm{A}$ $2.594 \AA$; $\mathrm{H} \cdots \mathrm{A} 1.864$ Å) between $\mathrm{H} 2$ and N2 atom, which stabilize the crystal structure $[11,12]$. In the crystal packing, dipole-dipole and van der Waals interactions are effective besides an intermolecular hydrogen bond in the molecular packing.

Author contributions: All the authors have accepted responsibility for the entire content of this submitted manuscript and approved submission.

Research funding: National Natural Science Foundation of China (grant no. 21861045) and Technology University Students Science and Technology Innovation and Entrepreneurship Training Program Project in Zunyi Medical University (No. ZYDC2020038).

Conflict of interest statement: The authors declare no conflicts of interest regarding this article.

\section{References}

1. Rigaku Corporation. CrysAlis ${ }^{\mathrm{PRO}}$; Yarnton, Oxfordshire, England, 2015.

2. Sheldrick G. M. SHELXTL - integrated space-group and crystalstructure determination. Acta Crystallogr. 2015, A71, 3-8.

3. Sheldrick G. M. Crystal structure refinement with SHELXL. Acta Crystallogr. 2015, C71, 3-8.

4. Dolomanov 0. V., Bourhis L. J., Gildea R. J., Howard J. A. K., Puschmann H. OLEX2: a complete structure solution, refinement and analysis program. J. Appl. Crystallogr. 2009, 42, 339-341.

5. Reiss H., Shalit H., Vershinin V., More N. Y., Forckosh H., Pappo D. Cobalt [salen]-catalyzed selective aerobic oxidative crosscoupling between electron-rich phenols and 2-naphthols. J. Org. Chem. 2019, 84, 7950-7960.

6. Parsons S., Flack H. D., Wagner T. Use of intensity quotients and differences in absolute structure refinement. Acta Crystallogr. 2013, B69, 249-259.

7. Schiff H. The syntheses and characterization of Schiff base. Ann. Chem. Pharm. Suppl. 1864, 3, 343-349.

8. Belokon Y. N., North M., Parsons T. Vanadium-catalyzed asymmetric cyanohydrin synthesis. Org. Lett. 2000, 2, 1617-1619.

9. Zeng X. P., Cao Z. Y., Wang X., Chen L., Zhou F., Zhu F., Wang C. H., Zhou J. Activation of chiral (salen)AlCl complex by phosphorane for highly enantioselective cyanosilylation of ketones and enones. J. Am. Chem. Soc. 2016, 138, 416-425.

10. Kunisu T., Oguma T., Katsuki T. Aerobic oxidative kinetic resolution of secondary alcohols with naphthoxide-bound iron(salan) complex. J. Am. Chem. Soc. 2011, 133, 12937-12939.

11. Jiao L., Tan H., Sun J., Zhang L., Wu Q. Crystal structure of rac-trans$\mathrm{N}, \mathrm{N}^{\prime}$-bis(3,5-dibromosalicylidene)-1,2-cyclohexanediamine, $\mathrm{C}_{20} \mathrm{H}_{18} \mathrm{Br}_{4} \mathrm{~N}_{2} \mathrm{O}_{2}$. Z. Kristallogr. NCS 2020, 235, 847-848.

12. Wu Q., Tang Y., Li J., Li Y., Fang Y. Crystal structure of rac-trans$N, N^{\prime}$-bis(3,5-diiodosalicylidene)-1,2-cyclohexanediamine, $\mathrm{C}_{20} \mathrm{H}_{18} \mathrm{I}_{4} \mathrm{~N}_{2} \mathrm{O}_{2}$. Z. Kristallogr. NCS 2019, 234, 69-70. 